\title{
Diagnose bradykarder Herzrhythmusstörungen
}

Carsten Walter Israel

Ist die Funktion des Sinusknotens oder die atrioventrikuläre Erregungsleitung gestört, kommt es zu einer Bradykardie - die Herzfrequenz verlangsamt sich. Je nach Symptomatik des Patienten kann eine Schrittmacher-Therapie indiziert sein. Doch die Diagnose einer Bradykardie ist oftmals kompliziert, die Methoden vielseitig: Sie reichen von einem einfachen Ruhe-EKG bis zum LangzeitMonitoring mit implantiertem Loop-Rekorder.

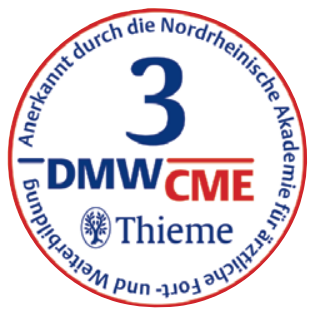

\section{Pathologische Bedeutung und Epidemiologie}

Definition I Eine Herzfrequenz $<60 /$ min ist per Definition eine Bradykardie [1].

Da diese Definition willkürlich ist und praktisch jeder gesunde Mensch zu bestimmten Zeiten (z. B. im Schlaf) eine Herzfrequenz unter 60 / min zeigt, ist eine Bradykardie an sich nicht pathologisch.

Bradykarde Herzrhythmusstörungen werden pathologisch, wenn sie zu Symptomen führen oder assoziiert sind mit

- einem erhöhten Risiko für einen Sturz,

- der Entwicklung einer Herzinsuffizienz oder

- einem plötzlichen Herztod.

In Deutschland werden wegen bradykarder Herzrhythmusstörungen jährlich über 100000 Herzschrittmacher implantiert [2, 3].

Anamnese I Bei der Diagnose einer Bradykardie muss man immer erst den Mechanismus erkennen (prognostisch relevant?) und einen klinischen Kontext herstellen:

- assoziierte Symptomatik?

- Sportler?

- vagaler Einfluss?

- reversible Ursachen?

Erst dann kann beurteilt werden, ob und wie eine Bradykardie einzustufen und zu behandeln ist.

\section{Formen von Bradykardien}

Einteilung | Bradykardien werden in Erregungsbildungsstörungen (Sick-Sinus-Syndrom) und atrioventrikuläre $(\mathrm{AV})$ bzw. ventrikuläre Erregungsleitungsstörungen eingeteilt. Alle weiteren Schrittmacher-Indikationen (z.B. Karotissinus-Syndrom, Vorhofflimmern mit bradykarder Überleitung) basieren auf diesen beiden Bradykardie-Mechanismen. $\mathrm{Zu}$ den verschiedenen Formen gehören:
- Erregungsbildungsstörungen:

Sick-Sinus-Syndrom

- Sinusbradykardie

- sinuatrialer Block, Sinusarrest

- Brady-Tachy-Syndrom

- chronotrope Inkompetenz

- Erregungsleitungsstörungen: AV-Block und Schenkelblock

- AV-Block ${ }^{\circ}$

- AV-Block II ${ }^{\circ}$ Typ Wenckebach (= Mobitz 1)

- AV-Block II $^{\circ}$ Typ Mobitz (= Mobitz 2)

> AV-Block II $^{\circ}$ mit 2:1-, 3:1- oder 4:1-Überleitung

- AV-Block III ${ }^{\circ}$

- AV-Block bei Vorhofflimmern

- Schenkelblockierungen

\section{Sick-Sinus-Syndrom}

Kranker Sinusknoten | Von einem Sick-Sinus-Syndrom spricht man, wenn durch eine SinusknotenFunktionsstörung Symptome ausgelöst werden. Eine asymptomatische Sinusbradykardie ist kein Sick-Sinus-Syndrom.

Schrittmacher sind nur indiziert, wenn Symptome vorliegen.

Im EKG werden 4 verschiedene Formen des SickSinus-Syndroms unterschieden:

(Permanente) Sinusbradykardie I Bei dieser Form des Sick-Sinus-Syndroms besteht eine Sinusbradykardie, erkennbar an einer P-Wellen-Frequenz $<60 / \mathrm{min}$.

Sinuatrialer (SA-) Block und Sinusarrest | Bei diesem Krankheitsbild werden Sinusknotenimpulse nicht gebildet bzw. nicht oder nur intermittierend auf den Vorhof übergeleitet. Die Impulse des Sinusknotens sind jedoch im KörperoberflächenEKG nicht sichtbar, sondern nur die P-Welle als Ausdruck der erfolgten Leitung vom Sinusknoten auf den Vorhof. Daher findet sich im EKG eine 
Pause ohne sichtbare P-Wellen (im Unterschied zum AV-Block, wo eine Pause durch nicht übergeleitete P-Wellen zu erkennen ist).

Ein Sinusarrest liegt vor, wenn innerhalb der physiologischen Variationsbreite des aktuellen Herzrhythmus keine P-Welle im EKG zu erkennen ist (meist für mehr als 2 Sekunden).

Es kann bei Sinusknoten-Stillstand zu einer Pause, aber auch zum Einspringen eines Ersatzrhythmus aus dem AV-Knoten mit schmalem QRS-Komplex ohne vorhergehende P-Wellen kommen. Dies nennt man ,junktionalen Ersatzrhythmus“ ( $\triangleright$ Abb. 1).

Brady-Tachy-Syndrom | Patienten mit Sick-SinusSyndrom haben oft auch Vorhofflimmern. Bei gesundem AV-Knoten wird Vorhofflimmern mit nur mäßiger Untersetzung auf den Ventrikel übergeleitet und führt zu einer Kammerfrequenz von etwa 220-Lebensalter, bei einem 70-Jährigen also von etwa $150 / \mathrm{min}$. Die Überleitung variiert durch vagale und sympathische Einflüsse, bewirkt aber insbesondere unter körperlicher Belastung eine Tachyarrhythmie.

Wenn das Vorhofflimmern spontan sistiert (paroxysmales Vorhofflimmern), kommt es beim Sick-Sinus-Syndrom wegen der Sinusknoten-Dysfunktion häufig zu einer längeren Pause, bevor der Sinusrhythmus wieder eintritt (präautomatische Pause) ( Abb. 2).

Oft bleibt der Sinusrhythmus auch im Weiteren bradykard. Ein Brady-Tachy-Syndrom als Unterform des Sick-Sinus-Syndroms ist daher oft hochsymptomatisch:

- Während der Phasen von Vorhofflimmern spüren die Patienten Symptome wie Herzrasen, Palpitationen oder Dyspnoe,

- bei einer präautomatischen Pause eine (Prä-) Synkope und

- während der nachfolgenden Sinusbradykardie Schwindel.

Die Phasen tachyarrhythmischer Überleitung bedürfen meist einer bradykardisierenden Medikation, die bei bekannten Pausen oder Bradykardien jedoch kontraindiziert ist. Deshalb ist das Brady-Tachy-Syndrom eine häufige Indikation zur Herzschrittmacher-Implantation.

Chrontrope Inkompetenz | Diese bezeichnet generell die Unfähigkeit, eine der körperlichen Belastung entsprechende Herzfrequenz bereitzustellen. Es existieren unterschiedliche formale Definitionen, die für eine therapeutische Entscheidung jedoch oft nicht hilfreich sind. Im klinischen Alltag hat sich die pragmatische Definition bewährt: Danach kann von einer klinisch relevanten chronotropen Sinusknoten-Inkom-
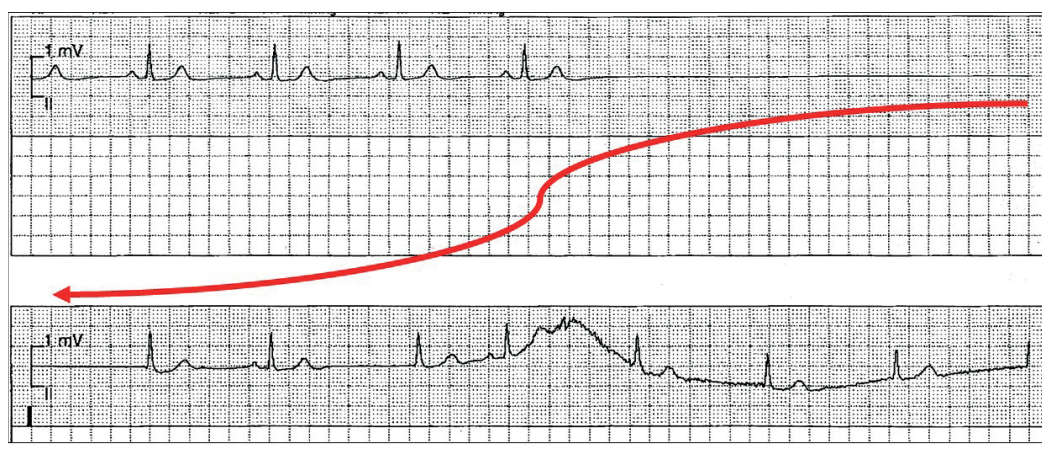

Abb. 1 Sinusarrest (fortlaufende Registrierung, roter Pfeil, $25 \mathrm{~mm} / \mathrm{s}$ ): Nach 4 Aktionen mit P-Wellen sistiert plötzlich die Sinusknotenaktivität, es folgt eine Asystolie für ca. 6,5s, die von einer Ventrikelaktion ohne vorausgehende P-Welle beendet wird (junktionaler Ersatzschlag). Auch im Weiteren treten neben einer Sinusaktion und einer supraventrikulären Extrasystole mehrere junktionale Ersatzschläge auf, der Sinusrhythmus hat sich noch nicht wieder erholt.

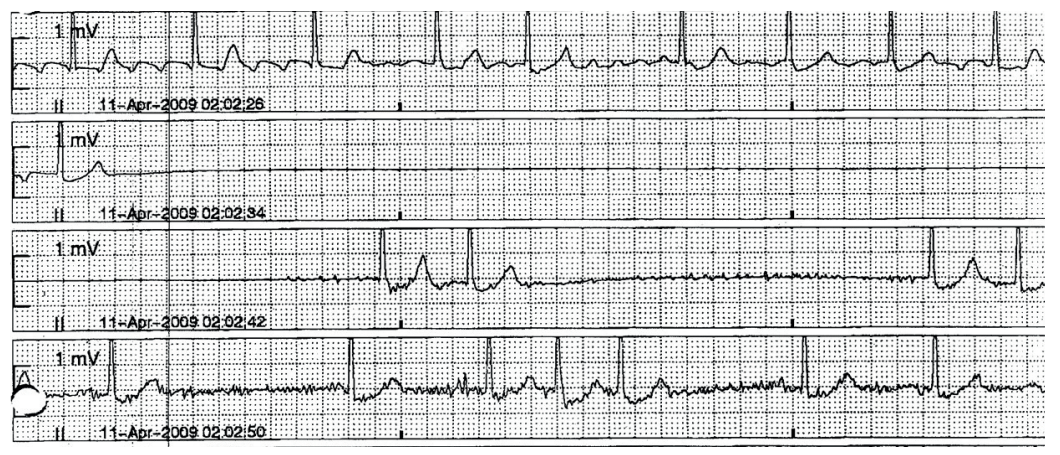

Abb. 2 Präautomatische Pause (fortlaufende Registrierung, $25 \mathrm{~mm} / \mathrm{s}$ ): Nach einer Phase von Vorhofflimmern / -flattern stoppt die Tachyarrhythmie spontan, es tritt jedoch zunächst kein Sinusrhythmus auf. Nach einer Asystolie von ca. 10,5 s (präautomatische Pause) treten ein junktionaler Ersatzschlag und eine supraventrikuläre Extrasystole gefolgt von einer weiteren Pause (Sinusarrest für weitere 3,3 s) auf. Erst im unteren Streifen ist wieder Sinusrhythmus zu sehen. Im Unterschied zum Arrest im engeren Sinne kann eine präautomatische Pause nur nach Sistieren eines anderen Rhythmus

(z. B. Vorhofflimmern / -flattern, Schrittmacher-Stimulation) auftreten und beschreibt die Zeit bis zum Eintreten eines Sinusrhythmus.

petenz ausgegangen werden, wenn an der anaeroben Schwelle (Spiroergometrie) oder im Langzeit-EKG bei Alltagsbelastung die maximale Herzfrequenz $<90-100 /$ min liegt. Diese dürfte durch Schrittmachertherapie besser werden.

\section{AV-Block}

Funktionell oder strukturell? | Die pathologische Bedeutung eines AV-Blocks hängt davon ab, ob er eine Folge extrinsischer Faktoren (funktionell: vagaler oder medikamentöser Einfluss) ist oder eine intrinsische (strukturell) Leitungsstörung vorliegt. Im Unterschied zur Sinusbradykardie kann eine Bradykardie infolge eines AV-Blocks eine prognostische Bedeutung haben, die man mit Schrittmachertherapie beeinflussen kann.

Daher kann ein AV-Block im Unterschied zum Sick-Sinus-Syndrom auch ohne Symptomatik eine Indikation zur Schrittmachertherapie sein. 


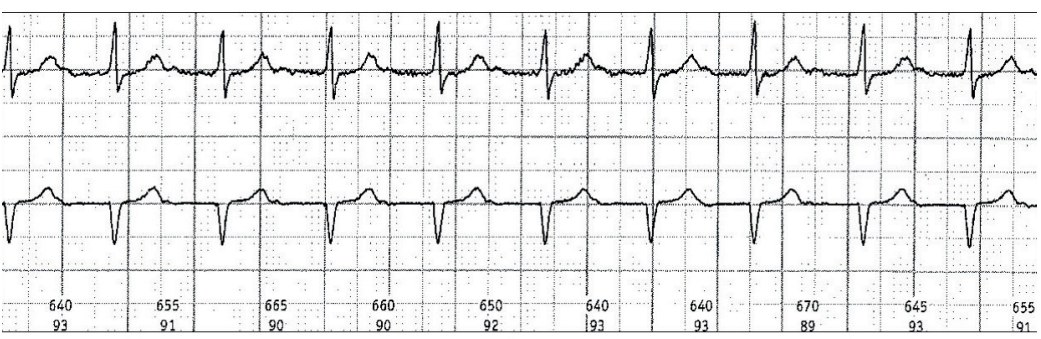

Abb. 3 Langer AV-Block I ${ }^{\circ}$ mit Pseudo-Schrittmacher-Syndrom (Schrittmacher-Syndrom ohne Schrittmacher) (2 Ableitungen, $25 \mathrm{~mm} / \mathrm{s}$, Langzeit-EKG-Registrierung): Bei körperlicher Belastung mit Anstieg der Herzfrequenz auf ca. 90 / min kommt es nicht zur Verkürzung eines vorbestehenden AV-Blocks $I^{\circ}$, der offenbar nicht vagal vermittelt, sondern fixiert ist. Hierdurch rückt die P-Welle an die T-Welle des vorausgehenden QRS-Komplexes (besser sichtbar in der unteren Ableitung). Dies kann eine „Vorhofpfropfung", eine Vorhofkontraktion gegen geschlossene AV-Klappen, hervorrufen und das Herzzeitvolumen reduzieren bzw. zu einer vasovagalen Reaktion führen.

AV-Block $I^{\circ}$ | Bei diesem werden alle P-Wellen übergeleitet, jedoch beträgt die PQ-Zeit>200 ms. Er ist meist funktionell durch vagalen Einfluss verursacht und verschwindet unter körperlicher Belastung. Eine intrinsische, nicht vagale Ursache kann vorliegen, wenn ein AV-Block I ${ }^{\circ}$ unter Belastung persistiert oder sogar länger wird. Eine $\mathrm{PQ}-$ Zeit $>300 \mathrm{~ms}$ ist bei rein vagalem Einfluss ungewöhnlich - in diesem Fall erfolgt eher der Übergang in einen AV-Block II $^{\circ}$ mit WeckebachPeriodik. Diese Form kann bei steigender Sinusfrequenz zu einem Pseudo-Schrittmacher-Syndrom führen (Vorhof-Pfropfungswellen bei einem Patienten ohne Schrittmacher): Bei diesem liegen die P-Wellen in der ST-Strecke oder T-Welle des vorausgehenden QRS-Komplexes ( Abb. 3).

AV-Block II ${ }^{\circ}$ Wenckebach I Bei diesem AV-Block verlängert sich die PQ-Zeit übergeleiteter P-Wellen periodisch bis eine P-Wellen-Überleitung ausfällt. Pathognomonisch ist, dass die erste übergeleitete P-Welle nach der Pause immer die kürzeste PQ-Zeit aufweist. Er ist meist vagal ausgelöst und verschwindet unter Belastung - die Überleitung verschlechtert sich nicht, wenn die Sinusfrequenz durch Atropin oder körperliche Belastung gesteigert wird.
AV-Block II Typ Mobitz I In diesem Fall fällt die Überleitung von P-Wellen sporadisch und ohne Periodik aus. Die PQ-Zeit übergeleiteter P-Wellen ist im Unterschied zum AV-Block II $^{\circ}$ Typ Wenckebach konstant. Meist ist diese Form im/unterhalb des His-Bündels lokalisiert und nicht vagal vermittelt. Die Überleitung verschlechtert sich unter Steigerung der Sinusfrequenz durch Atropin oder körperliche Belastung.

AV-Block III $^{\circ}$ | Beim AV-Block III $^{\circ}$ sind atriale und ventrikuläre Aktionen per definitionem unabhängig voneinander. Die Vorhoffrequenz ist daher ungleich und kein Vielfaches der Ventrikelfrequenz. Um einen AV-Block III $^{\circ}$ von einem AV-Block $\mathrm{II}^{\circ}$ mit 2:1- (oder 3:1-) Überleitung zu unterscheiden, werden oft zahlreiche Ventrikelaktionen benötigt - optimal mit einer Schreibgeschwindigkeit von $25 \mathrm{~mm} / \mathrm{s}$ und einem langen Rhythmusstreifen. Bei einem AV-Block III ${ }^{\circ}$ liegt im Ventrikel in der Regel ein Ersatzrhythmus vor, entweder

- aus dem Bereich der AV-Junktion bzw.

- aus dem His-Bündels (Dauer des QRS-Komplexes $\leq 120 \mathrm{~ms}$ ) oder

- von infrahisär (QRS-Dauer > $120 \mathrm{~ms}$ ) aus einem der Tawara-Schenkel bzw. Purkinje-Fasern.

Generell ist der Ersatzrhythmus umso distaler lokalisiert, je langsamer er ist und je breiter der QRS-Komplex.

AV-Block II ${ }^{\circ}$ mit 2:1- oder 3:1-Block | Bei einem solchen AV-Block wird nur jede 2. oder 3. P-Welle übergeleitet. Er zählt zu den höhergradigen AVBlockierungen. Der Unterschied zu einem AVBlock III $^{\circ}$ mit stabilem Ersatzrhythmus wird oft erst in einem längeren Rhythmusstreifen deutlich (Schreibgeschwindigkeit von $25 \mathrm{~mm} / \mathrm{s}$ ).

Paroxysmaler AV-Block I Bei einem paroxysmalen AV-Block besteht eine normale 1:1-AV-Überleitung (ggf. mit AV-Block $I^{\circ}$ oder Schenkelblock) bis plötzlich konsekutive P-Wellen nicht mehr übergeleitet werden. Ein Ersatzrhythmus fehlt in der Regel. Oft leitet eine supraventrikuläre oder
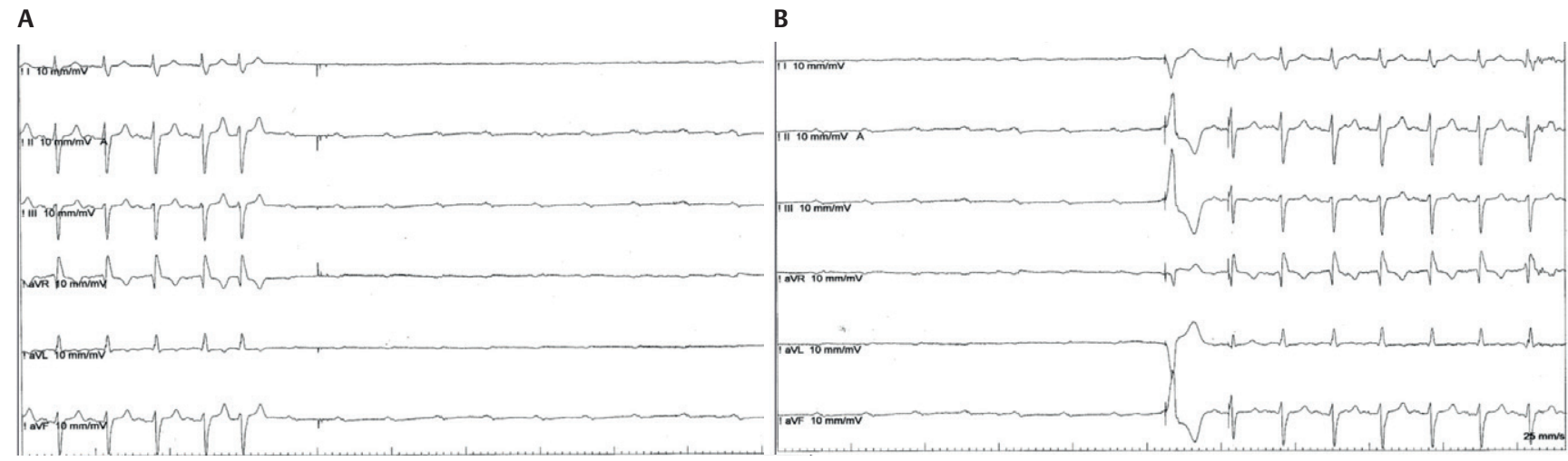

Abb. 4 Paroxysmaler kompletter AV-Block: Extremitäten-Ableitungen, 25 mm / s. Nach 4 übergeleiteten Sinusaktionen (AV-Block I, Rechtsschenkelblock) kommt es zu einer supraventrikulären Extrasystole (5. Aktion) mit nachfolgendem komplettem AV-Block (A). Da auch nach ca. 20 Aktionen keine Eigenüberleitung zurückkehrt, wird über die soeben implantierte Ventrikelelektrode ein Stimulus abgegeben. Hiernach besteht wieder 1:1-Überleitung (1 Schlag mit Pseudofusion) (B). Mechanismus: Phase IV-Block. 
ventrikuläre Extrasystole einen paroxysmalen AV-Block ein und beendet diesen. Der Mechanismus eines paroxysmalen AV-Block III $^{\circ}$ besteht in der Regel in einem Phase IV-Block der infranodalen AV-Überleitung: Ein verkürztes RR-Intervall (typischerweise durch eine Extrasystole) führt dazu, dass das Ruhemembran-Potenzial so gering wird, dass das Leitungsgewebe unerregbar und die P-Wellen nicht mehr übergeleitet werden ( Abb. 4).

AV-Block bei Vorhofflimmern | Hierbei handelt es sich um einen Sonderfall. Erkennbar ist er an regelmäßigen R-R-Abständen, oft mit breitem QRS. Ein regelmäßiger Kammerrhythmus beweist das Vorliegen eines (anhaltenden) AV-Blocks $\mathrm{III}^{\circ}$, da Vorhofflimmern immer unregelmäßig überleitet $(\checkmark$ Abb. 5). Dies ist eine Schrittmacher-Indikation. Für die viel häufiger vorkommende unregelmäßige Bradykardie bei Vorhofflimmern ist der Grad der AV-Blockierung nicht definiert, auch wenn für Pausen $>3 \mathrm{~s}$ ein intermittierender AVBlock III $^{\circ}$ angenommen werden kann. Wie der Sinusrhythmus unterliegt auch die AV-Überleitung einem vagalen Einfluss. Intermittierende, unregelmäßig bradykarde Phasen bei Vorhofflimmern nachts sowie in Ruhephasen und Tachyarrhythmie bei körperlicher Aktivität sind daher häufige, normale Befunde bei Patienten mit Vorhofflimmern.

Vorhofflimmern mit intermittierender, unregelmäßig bradykarder Kammerantwort ist nur dann eine Schrittmacher-Indikation, wenn

- Symptome vorliegen oder

- eine bradykardisierende Medikation zur Frequenzkontrolle der tachykarden Phasen notwendig ist.

Zur Differenzialdiagnose gehört das Vorhofflattern, bei dem regelmäßige R-R-Abstände normal sind.

\section{Schenkelblockierungen}

Linksschenkelblock immer pathologisch | Da der rechte Tawara-Schenkel physiologischerweise etwas langsamer als der linke leitet, ist ein inkompletter Rechtsschenkelblock meist ohne Krankheitswert (z.B. bei Jugendlichen). Auch ein kompletter Rechtsschenkelblock hat nicht immer eine pathologische Bedeutung.

Ein Linksschenkelblock ist dagegen immer auffällig bzw. pathologisch. Er geht jedoch nicht per se mit einer Bradykardie einher, solange der rechte Schenkel leitet.

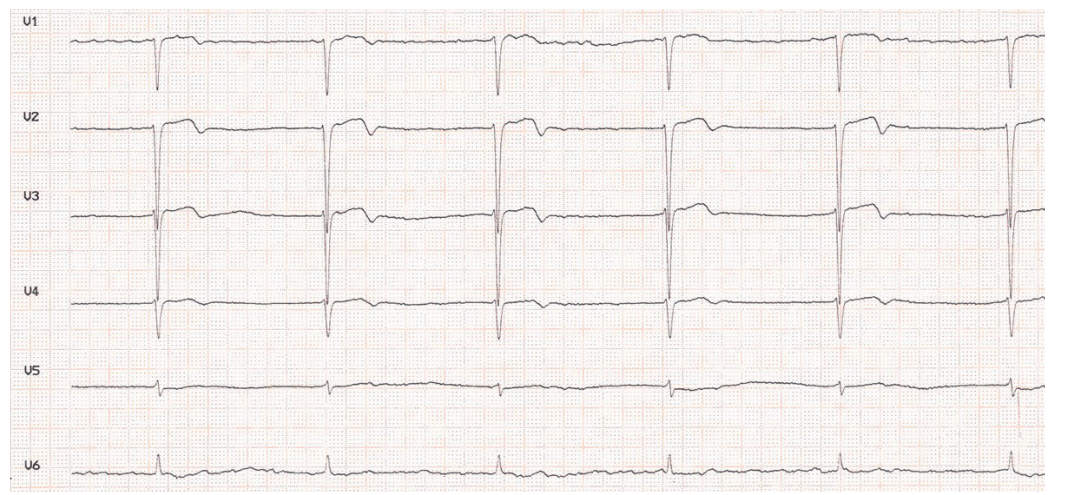

Abb. 5 AV-Block bei Vorhofflimmern (Brustwandableitungen, $25 \mathrm{~mm} / \mathrm{s}$ ): In Ableitung V1 sind Vorhofflimmer-Aktionen zu erkennen, P-Wellen fehlen. Der regelmäßige ventrikuläre Rhythmus (hier bei 35 / min) beweist bei Vorhofflimmern einen AV-Block III․

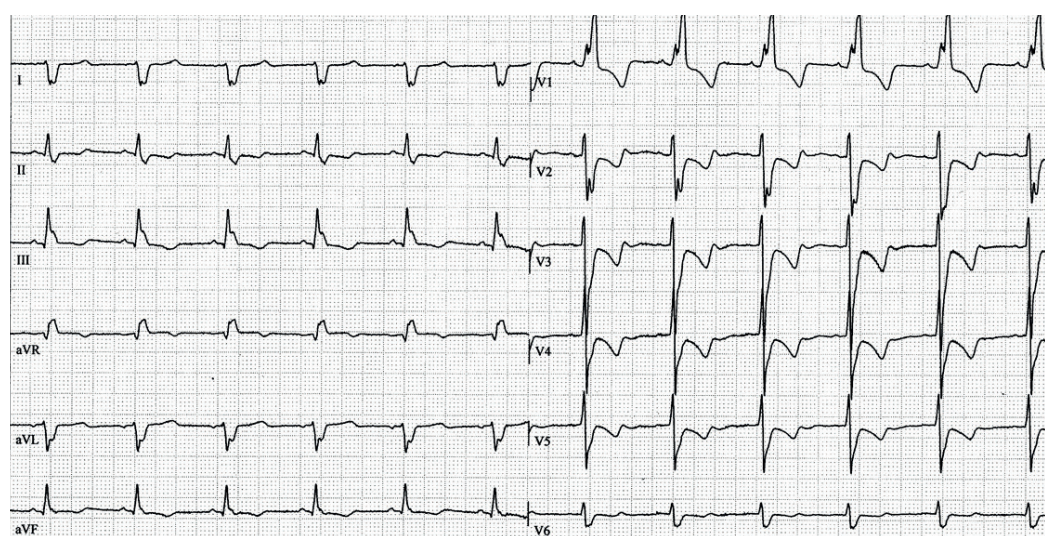

Abb. 6 Bifaszikulärer Block in Form von Rechtsschenkelblock und linksposteriorem Hemiblock ( $25 \mathrm{~mm} / \mathrm{s}$ ): Die dominante, breite, doppelgipflige R-Zacke in V1 zeigt einen Rechtsschenkelblock an. Zusätzlich fallen ein Rechtslagetyp, eine breite / tiefe S-Zacke in I, ein sehr schmales Q und eine Knotung in Ableitung III auf, die einen linksposterioren Hemiblock definieren.

Von Interesse bei Patienten mit Verdacht auf rhythmogene Synkope sind Schenkelblockierungen, die einen (infrahisären) kompletten AVBlock vorhersagen.

Bifaszikuläre Blockierungen | Neben der Leitungsunterbrechung beider linker Faszikel (Linksschenkelblock) findet sich am häufigsten die Kombination eines

- kompletten Rechtsschenkelblocks mit

- einem linksanterioren Hemiblock.

Dieser ist an einem überdrehten Linkslagetyp mit tiefem $S$ in V6 zu erkennen. Seltener findet sich eine Kombination zwischen komplettem Rechtsschenkelblock und linksposteriorem Hemiblock. In Abb. 6 findet sich eine QRS-Achse $\geq 90^{\circ}$ (Rechtslagetyp oder überdrehter Rechtslagetyp). In Ableitung I zeigt sich ein sehr breites $S$, in Ableitung III ein sehr schmales $(<40 \mathrm{~ms}) \mathrm{Q}$ und eine Knotung im absteigenden Schenkel der RZacke. 


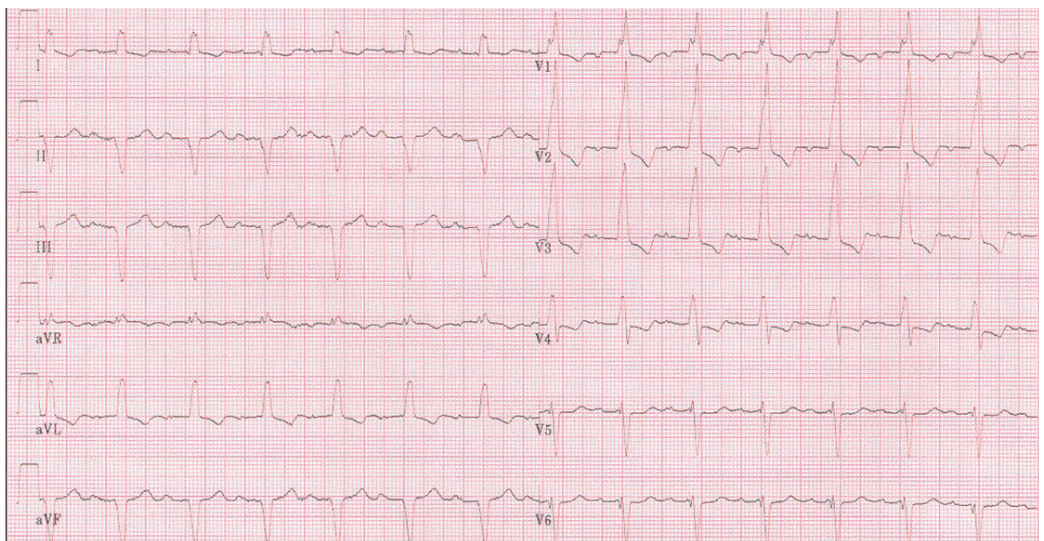

Abb. 7 Bifaszikulärer Block und AV-Block $I^{\circ}$ mit Synkope (intermittierender trifaszikulärer Block, $25 \mathrm{~mm} / \mathrm{s}$ ): Bei einem älteren Patienten mit Synkope fallen neben einem Rechtsschenkelblock (dominantes R in V1) ein linksanteriorer Hemiblock (überdrehter Linkstyp, tiefes S in V6) und ein AV-Block I $^{\circ}$ mit einer PQ-Zeit von 240 ms auf. In diesem Fall ist von einer Leitungsverzögerung im linksposterioren Schenkel auszugehen, bei dessen Ausfall ein trifaszikulärer Block die Synkope erklärt.

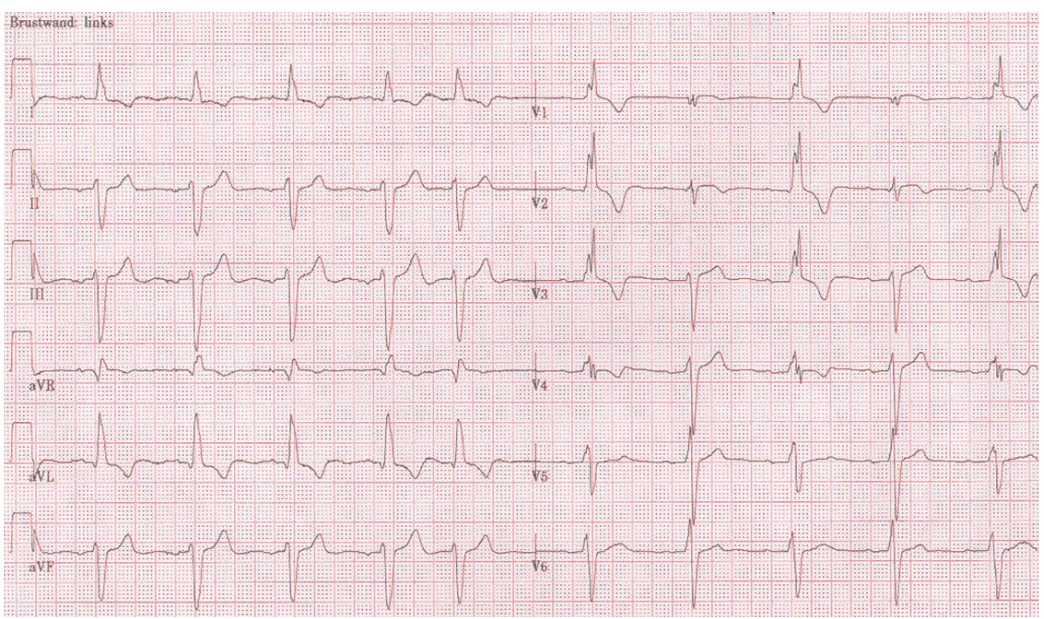

Abb. 8 Drohender trifaszikulärer Block ( $25 \mathrm{~mm} / \mathrm{s}$ ): Bei dieser Patientin mit Synkope liegt ein bifaszikulärer Schenkelblock (Rechtsschenkelblock und linksanteriorer Hemiblock) vor. In den Brustwandableitungen fällt auf, dass sich bei jeder 2. Aktion die PQ-Zeit von 240 auf 280 ms verlängert und sich gleichzeitig die Morphologie des nachfolgenden QRS-Komplexes ändert (keine Rechtsschenkelblock-Morphologie mehr). Es ist davon auszugehen, dass bei den Aktionen mit einer PQ-Zeit von 240 ms der linksposteriore Schenkel noch leitet, diese Leitung sich jedoch erschöpft und sich daher bei jedem 2 . Zyklus die PQ-Zeit auf 280 ms verlängert. Zu diesem Zeitpunkt wird die Leitung vom rechten Schenkel übernommen (Rechtsschenkelblock verschwindet). Hier sind offenbar alle 3 Schenkel erkrankt und leiten gar nicht (linksanteriorer Schenkel), nur intermittierend (linksposteriorer Schenkel) oder nur verzögert (rechter Schenkel).

Bifaszikuläre Blockierungen

- Kompletter Linksschenkelblock:

> QRS> $120 \mathrm{~ms}$ (typisch: > $140 \mathrm{~ms}$ ), fehlendes (sehr kleines) $r$ mit tiefem $S$ in V1, dominantes R in V6 mit Knotung

- Rechtsschenkelblock + linksanteriorer Hemiblock:

> QRS> $120 \mathrm{~ms}$, R in V1 (M-förmig, rSR“ oder rsR'), überdrehter Linkstyp, S in V6

- Rechtsschenkelblock + linksposteriorer Hemiblock:

> QRS> $120 \mathrm{~ms}$, R in V1 (M-förmig, rSR' oder rsR'), QRS-Achse $>90^{\circ}, \mathrm{S}$ in I, schmales $Q$ und Knotung in III
Drohender trifaszikulärer Block | Wenn zu einem bifaszikulären Block ein AV-Block I ${ }^{\circ}$ hinzukommt, liegt der Verdacht nahe, dass diese AV-Verzögerung nicht im AV-Knoten, sondern im letzten noch leitenden Faszikel entsteht ( $\bullet$ Abb. 7). Dies gilt auch für einen kompletten Linksschenkelblock mit zusätzlichem AV-Block I ${ }^{\circ}$ (oder sogar $\mathrm{II}^{\circ}$ ), der nicht nur im AV-Knoten, sondern auch im rechten Schenkel lokalisiert sein kann. Dieser Verdacht erhärtet sich, falls anamnestisch Synkopen hinzukommen. Persistiert oder schreitet der AV-Block $\mathrm{I}^{\circ}$ unter Belastung/Sinusfrequenz-Steigerung fort, ist dies ein weiteres Argument für eine Leitungsverzögerung im letzten leitenden Faszikel. Beweisend ist letztlich eine elektrophysiologische Untersuchung, die eine verlängerte Leitung vom His-Bündel auf den Ventrikel (HVZeit) nachweist.

Alterniernder Schenkelblock | Werden bei einem Patienten neben Phasen eines Linksschenkelblocks auch Phasen eines Rechtsschenkelblocks registriert, liegt ein alternierender Schenkelblock vor. Dieser kann mit einem AV-Block III $^{\circ}$ gleichgesetzt werden: Beide Schenkel weisen fortgeschrittene Leitungsstörungen auf. Bei Progredienz der Leitungsstörung im einen Schenkel springt der andere (zuvor stärker blockierte) ein. Die Schenkelblockmorphologie kann von einem zum nächsten Schlag oder auch in konsekutiven EKGs wechseln ( Abb. 8).

\section{Extrinsisch induzierte Bradykardien}

Äußere Einflüsse | Neben einer intrinsischen strukturellen Erregungsbildungs- oder -leitungsstörung kann auch ein reversibler extrinsischer Einfluss eine Bradykardie verursachen. Beispiele hierfür sind vagale Einflüsse und Auswirkungen von Reflexkreisen. Es besteht eine normale intrinsische Sinus- / AV-Knoten-Funktion. Die Diagnose wird gestellt, wenn Sinusfrequenz und AVÜberleitung gleichzeitig langsamer werden.

Erhöhter Parasympathiko-Tonus | Bei erhöhtem Parasympathikotonus (z.B. Schlaf, vasovagale Reaktion) kann es sowohl zu einer Sinusbradykardie als auch zu einer AV-Blockierung kommen. Auch beides gleichzeitig ist möglich. Da der parasympathische Einfluss mit zunehmendem Lebensalter abnimmt, sind vagal vermittelte Bradykardien vor allem bei Patienten < 40 Jahre häufig. Neben der Anamnese (Auftreten zu Tageszeiten oder bei Situationen mit vorherrschendem Vagotonus) fällt bei vagal vermittelter AV-Blockierung eine reduzierte Sinusfrequenz auf.

Karotis-Sinus-Syndrom | Tätigkeiten wie Rasieren, Schlipsknoten, Kragenschließen, rasche oder starke Kopfdrehung z. B. beim Überholvorgang im Auto reizen die Barorezeptoren im Bereich der Karotis-Gabel. Dadurch kann ein Reflex ausgelöst 
werden, der einen Blutdruckabfall und / oder eine Frequenzreduktion bis hin zur Asystolie $>3 \mathrm{~s}$ bewirkt. Die Asystolie kann in einem Sinusarrest, einem AV-Block III $^{\circ}$ oder beidem bestehen.

\section{Ursachen}

Sick-Sinus-Syndrom I Die zugrundeliegende Pathologie des Sick-Sinus-Syndroms ist eine Fibrose im Bereich des Sinusknotens und seiner angrenzenden Strukturen. Diese kann zu einem Ausfall der Pacemaker-Zellen oder einer Blockierung der Leitung aus dem Sinusknoten in den Vorhof führen. Risikofaktoren für die Entwicklung eines Sick-Sinus-Syndroms sind in erster Linie

- Alter,

- arterielle Hypertonie,

- Erkrankungen mit Rechtsherzbelastung (z.B. COPD, Emphysem) und

- Herzinsuffizienz.

In Langzeit-Beobachtungen sind mit dem Syndrom außerdem folgende Ursachen assoziiert worden:

- verlängerte QRS-Dauer

- Rechtsschenkelblock

- niedrige Herzfrequenz

- Adipositas

- Größe

- Zeichen der Niereninsuffizienz

- Diabetes

- Anamnese kardiovaskulärer Ereignisse [4]

Vorhofflimmern I Sick-Sinus-Syndrom und Vorhofflimmern haben oft eine gemeinsame Ursache, die atriale Fibrose. Sie interagieren auf mehreren Ebenen miteinander: Vorhofflimmern unterdrückt die Sinusknotenaktivität mit der Folge eines Remodellings des Sinusknotens.

Eine Sinusbradykardie ist zumindest bei einigen Patienten ein Trigger für Vorhofflimmern.

AV-Blockierungen | Diese können bereits kongenital vorliegen infolge

- kongenitaler Vitien mit Einbeziehung der AVRegion (Ventrikelseptumdefekt, atrioseptaler Defekt vom Primumtyp etc.),

- Autoimmunerkrankungen der Mutter (v.a. Lupus erythematodes),

- Myokarditis oder

- idiopathisch.

Ein erworbener AV-Block ist häufig ischämisch verursacht (chronischer oder akuter Myokardinfarkt).

Neben Myokarditiden, Kardiomyopathie, Hypertonie und operativ-mechanischer Schädigung des
Reizleitungssystems - etwa nach Aortenklappenoperation oder transarteriellem Aortenklappenersatz - bleibt die Ursache bei ca. $40 \%$ der Patienten unklar (idiopathisch).

Reversibler AV-Block | Besonders AV-Blockierungen können zahlreiche reversible Ursachen haben: In erster Linie sollten virale, bakterielle und andere Infektionen ausgeschlossen werden, die eine akute Myokarditis verursachen. Auch nach einem Erythema nodosum (Borreliose mit LymeDisease, Sarkoidose) sollte gesucht bzw. gefragt werden. Selten kann es zu einem AV-Block im Rahmen von Sarkoidose, Kollagenose oder kardialen Metastasen kommen. Verschiedene neuromuskuläre Erkrankungen können Schenkel- und AV-Blockierungen verursachen. Bei Bradykardien sollte man Elektrolyte und bradykardisierende Medikamente kontrollieren. Eine Übersicht der Ursachen des Sick-Sinus-Syndroms und der AVBlockierungen finden Sie online ( sTab. 1).

\section{Symptomatik}

Intermittierende Bradykardien | Die neuen Leitlinien zur Schrittmachertherapie der Europäischen Gesellschaft für Kardiologie unterscheiden Symptome, die typisch für intermittierende und permanente Bradykardien sind (ESC, [5-7]). Intermittierende Bradykardien (Sinusarrest, paroxysmaler AV-Block) gehen mit Pausen und plötzlichen Frequenzabfällen einher. Sie führen zu den Kardinalsymptomen

- Synkope,

- Präsynkope und

- Schwindelattacken sowie zu

- plötzlichen Sehstörungen („Schwarzwerden vor Augen“),

- aufsteigender Hitze und

- Herzklopfen.

Permanente Bradykardien | Diese Bradykardien (Sinusbradykardie, 2:1-AV-Block) gehen häufig mit dauerhaft langsamer Herzfrequenz und daher mit Symptomen einer Herzinsuffizienz einher:

- Dyspnoe, insbesondere unter Belastung

- Ödeme

- generelle Belastungsintoleranz

- allgemeine Müdigkeit

- allgemeiner Schwindel und

- Orthostase

Unspezifische Symptome I Neben diesen eher typischen Symptomen bradykarder Herzrhythmusstörungen können auch sehr unspezifische Beschwerden bestehen. Dazu gehören besonders allgemeine kognitive Defizite wie

- Konzentrationsschwierigkeiten,

- kognitive Verlangsamung,

- Depression und

- Vergesslichkeit. 


$\begin{array}{ll}\text { Symptomhäufigkeit } & \text { EKG-Methode } \\ \text { ständig } & \text { Ruhe-EKG } \\ \text { täglich } & \text { 24h-Langzeit-EKG, stationäre Telemetrie } \\ \text { alle 2-3 Tage } & \text { 48-72 h-Langzeit-EKG, stationäre Telemetrie } \\ \text { einmal pro Woche } & \text { 7-Tage-Langzeit-EKG, Event-Rekorder } \\ \text { einmal pro Monat } & \text { Event-Rekorder (14-30 Tage) } \\ \text { weniger als einmal pro Monat } & \text { implantierbarer Loop-Rekorder }\end{array}$

Tab. 1 Die Art der EKGDiagnostik hängt von der Symptomhäufigkeit ab.
Minuten vor und nach Aktivierung manuell speichern. Daneben bestehen auch automatische Algorithmen zur Detektion und Speicherung des EKGs von Brady- und Tachyarrhythmien. Die Systeme haben in der Regel eine Laufzeit von 3-5 Jahren. Sie ermöglichen in einem Zeitraum von 4 Jahren bei ca. $80 \%$ der Patienten mit Synkopen, die nach konventionellem Work-up unklar bleiben, eine eindeutige Diagnose mit EKG-Symptom-Korrelation.

Spiroergometrie und Ergometrie | Einige Bradykardien können in Provokationstests oder invasiv nachgewiesen werden. Die Diagnose einer chronotropen Inkompetenz wird am besten mittels Spiroergometrie gestellt: Die anaerobe Schwelle und maximale Sauerstoffaufnahme sind damit objektiv messbar. So kann bei körperlicher Erschöpfung geklärt werden, ob dies kardiopulmonale oder nicht-kardiale Ursachen hat - oder ob es an Motivation mangelt.

Bei einer Herzfrequenz $<90-100 /$ min an der anaeroben Schwelle ist eine chronotrope Inkompetenz klinisch relevant und mit einer Schrittmacherstimulation verbesserbar.

Die Schreibweise auf 25 mm / s mit Rhythmusstreifen vereinfacht die Analyse, insbesondere eine Periodik und ein Grundrhythmus können oft erst nach mehreren Zyklen beurteilt werden.

EKG nach Symptomhäufigkeit | Bei intermittierenden Bradykardien ist es notwendig, die Monitoringphase der Symptomhäufigkeit anzupassen ( Tab. 1). Treten Symptome täglich auf, sind ein 24-Stunden-Langzeit-EKG oder eine stationäre Telemetrie in der Regel ausreichend. Um eine Rhythmus-Symptom-Korrelation herzustellen, ist die Dokumentation der Uhrzeit der Symptome durch den Patienten entscheidend. Die Monitoring-Phase durch konventionelles kontinuierliches Langzeit-EKG bzw. Telemetrie kann auf 48-72 h verlängert werden, wenn die Beschwerden etwas seltener auftreten (z. B. alle 2-3 Tage). Bei geringerer Symptomfrequenz (z.B. 1 Mal pro Woche bis 1 Mal pro Monat) kann die Anlage eines Event-Rekorders bzw. externen Loop-Rekorders sinnvoll sein. Damit kann der Patient täglich selbst die Elektroden wechseln und mittels Knopfdruck bei Symptomen eine EKG-Speicherung triggern.

Bei vielen Patienten treten Symptome deutlich seltener als einmal pro Monat auf, z. B. eine Synkope.

Loop-Rekorder I In diesem Fall besteht die Möglichkeit der Implantation eines Loop-Rekorders (z.B. BioMonitor ${ }^{\circledR}$, Fa. Biotronik, Reveal ${ }^{\circledR}$, Fa. Medtronic oder Confirm ${ }^{\circledR}$, Fa. St. Jude Medical). Der Patient kann bei Symptomen ein EKG für einige
Ersatzweise kann eine chronotrope Inkompetenz auch in einer Ergometrie auf dem Fahrrad oder Laufband beurteilt werden. Bei fehlender Belastbarkeit (z.B. Knie-/Hüftprobleme) ist auch ein Langzeit-EKG möglich, sofern der Patient sich hierunter körperlich belastet.

Kipptisch-Untersuchung | Bei neurokardialer Synkope kann eine Kipptisch-Untersuchung eine Bradykardie oder Asystolie provozieren. Die Aussagekraft ist jedoch eingeschränkt: Auch bei Patienten mit klinischer neurokardialer Synkope vom Asystolie-Typ kann eine Reaktion ausbleiben oder der Patient reagiert lediglich vasodepressiv mit einem Blutdruckabfall.

Karotissinus-Massage I Bei Verdacht auf einen hypersensitiven Karotissinus kann eine Karotissinus-Massage hilfreich sein. Dies ist jedoch nur sinnvoll, wenn die Synkope im zeitlichen Zusammenhang mit einer Karotisreizung im Alltag steht (Krawattebinden, Rasieren, rasche/heftige Kopfdrehung). Denn ein beträchtlicher Anteil älterer Menschen zeigt auf Karotisdruck eine völlig unspezifische Reaktion in Form von Herzfrequenzabfall oder Asystolie und nach der wahren Ursache einer Synkope (z.B. Kammertachykardien) wird nach einem falsch-positiven Karotis-Druckversuch nicht weiter gesucht. Vor einem KarotisDruckversuch sollte die Karotis auskultiert und bei Strömungsgeräusch mit Farbduplex untersucht werden. So kann man eine Plaqueablösung mit Schlaganfall durch Karotisdruck vermeiden, auch wenn die Evidenz hierfür eher anekdotisch ist. 
Infrahisäre Leitung messen | Bei bifaszikulärem (inklusive Linksschenkel-) Block oder AV-Block I ${ }^{\circ}$ mit PQ> 300 ms kann es bei Schrittmacher-Indikation wegen unklarer Synkope hilfreich sein, die infrahisäre Leitung (HV-Zeit) zu messen.

\section{Provokationstest in der Bradykardie- Diagnostik}

- Fahrradergometrie (chronotrope Inkompetenz, Verschlechterung der Überleitung bei infrahisärem AV-Block)

- Kipptisch-Untersuchung (neurokardiale Synkope?)

- Karotis-Druckversuch (nur bei Synkopen im Zusammenhang mit Karotisreizung im Alltag)

- elektrophysiologische Untersuchung (infrahisäre Leitungsstörungen, Auslösung ventrikulärer Arrhythmien in der SynkopenDifferenzialdiagnose)

\section{Synkopenursache unklar}

Elektrophysiologie I Manche Synkopen bleiben trotz Abklärung möglicher Ursachen unklar (inkl. Implantation eines Loop-Rekorders). Eine invasive elektrophysiologische Untersuchung (EPU) ist jedoch nur indiziert, wenn im EKG Hinweise für eine mögliche Bradykardie/Pause vorhanden sind (z. B. Schenkelblock, AV-Block $\mathrm{I}^{\circ}$ oder $\mathrm{II}^{\circ}$ ). Indiziert ist eine EPU auch, wenn in der chronischen Postinfarktphase nicht-anhaltende Kammertachykardien und eine mäßige linksventrikuläre Funktionsminderung (EF 36-45\%) den Verdacht auf maligne ventrikuläre Rhythmusstörungen lenken. Die EPU erübrigt sich, wenn gleichzeitig die Indikation zur primärprophylaktischen ICDTherapie besteht. Eine Schrittmacher-Implantation ist bei Synkope oder unklarem Sturz nicht sinnvoll, wenn keine Bradykardie bzw. Leitungsstörung nachgewiesen wurde.

Vorgehen I Mögliches Prozedere bei unklarer Synkope und (Links-) Schenkelblock:

- Abwarten (jüngerer Patient, geringes Verletzungsrisiko)

- Implantation eines Loop-Rekorders

- elektrophysiologische Untersuchung (z.B. bei gleichzeitigem AV-Block ${ }^{\circ}$ )

Bei weiter negativen Testergebnissen ggf. im Einzelfall:

- DDD-Schrittmacher-Implantation (hohes Verletzungsrisiko, älterer Patient)

- CRT-Schrittmacher-Implantation (linksventrikuläre Ejektionsfraktion 36-45\%)

- CRT-Defibrillator-Implantation (linksventrikuläre Ejektionsfraktion $\leq 35 \%$ )

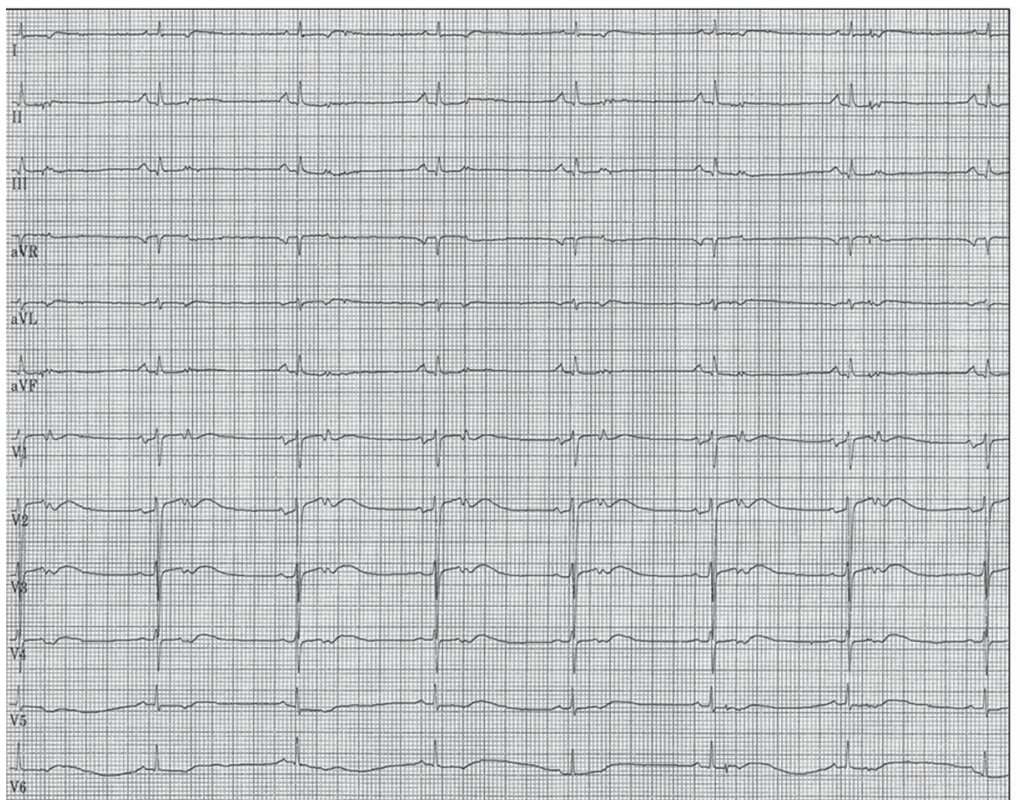

Abb. 9 Blockierter supraventrikulärer Bigeminus: Papiergeschwindigkeit $25 \mathrm{~mm} / \mathrm{s}$. Auf den ersten Blick (z. B. Ableitung II) scheint eine Sinusbradykardie um 44 / min vorzuliegen. Bei Analyse der Ableitungen V1-V3 fällt jedoch eine Veränderung in der ST-Strecke auf, die supraventrikulären Extrasystolen nach jeder Sinusaktion entspricht (supraventrikulärer Bigeminus). Die supraventrikulären Extrasystolen fallen so früh ein, dass der AV-Knoten noch refraktär ist (blockierter supraventrikulärer Bigeminus).

\section{Differenzialdiagnose im EKG}

Bei EKG beachten | Einige Fallstricke bestehen bei der EKG-Diagnose von Bradykardien. Entscheidend für eine korrekte Diagnose ist oft

- die Verfügbarkeit eines 12-Kanal-EKGs und nicht nur einzelner Ableitungen,

- eine Schreibweise bei $25 \mathrm{~mm} / \mathrm{s}$, durch die PWellen wesentlich besser sichtbar werden, und - ein Rhythmusstreifen, der längere Perioden erst sichtbar macht (z.B. zunehmende Verlängerung des PQ-Intervalls über 6-7 Zyklen mit periodischem Ausfall der Überleitung alle $8 \mathrm{Zy}$ klen).

SA-Block oder supraventrikuläre Extrasystole (SVES) mit postextrasystolischer Pause? I In einigen EKGs ist es schwierig zu unterscheiden, ob eine Pause ohne sichtbare P-Welle durch Ausfall einer Sinusaktion oder durch eine oder mehrere SVES mit postextrasystolischer Pause zustande kommt. Hier kann die Messung des PP-Intervalls helfen: Bei einem SA-Block nimmt dieses kontinuierlich ab (typischerweise 10-40 ms zwischen 2 Zyklen), bevor es zu einer Pause kommt. SVES oder ein supraventrikulärer Bigeminus zeigen diese Periodik nicht.

Sinusbradykardie oder blockierte SVES? I Im 1oder 2-Kanal-EKG (Langzeit-EKG, TelemetrieStreifen) kann sich eine Bradykardie manchmal als Sinusbradykardie zeigen, die sich im 12-Kanal-EKG als blockierte supraventrikuläre Extrasystolie herausstellt. Hier hilft manchmal der Vergleich der ST-Strecke/T-Welle zwischen nor- 


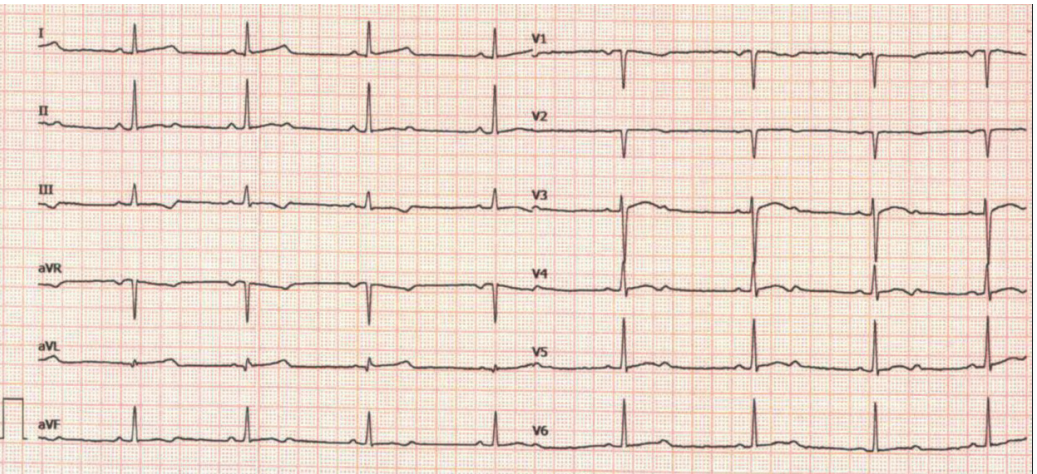

Abb. 10 Long-QT-Syndrom Typ 2 ( $25 \mathrm{~mm} / \mathrm{s}$ ): Bradykardie um 47 / min. In den Ableitungen II und V3-V5 finden sich doppelgipflige T-Wellen, die typisch für das Long-QT-Syndrom Typ II sind. Die Differenzialdiagnose umfasst die Sinusbradykardie und einen blockierten supraventrikulären Bigeminus.

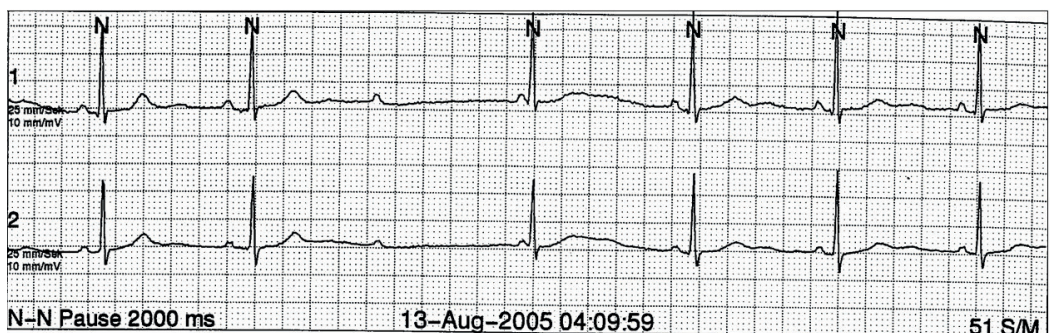

Abb. 11 Vagal nicht-übergeleitete P-Welle ( $25 \mathrm{~mm} / \mathrm{s}$, 2-Kanal-Langzeit-EKG): Um kurz nach 4 Uhr nachts kommt es zu einer nicht übergeleiteten P-Welle. Diese P-Welle tritt etwas später auf als die vorhergehende (Verlängerung der Sinuszykluslänge von 1040 auf $1080 \mathrm{~ms}$ ). Die gleichzeitige Verlangsamung von Sinusknoten- und AV-KnotenAktivität spricht für einen externen Einfluss, der beide Zentren beeinflusst (vagaler Einfluss).

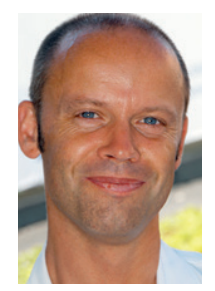

PD Dr. med. Carsten Walter Israel

ist ist Chefarzt der Klinik für Innere Medizin - Kardiologie, Diabetologie und Nephrologie des Evangelischen Krankenhauses Bielefeld. C.W.Israel@ em.uni-frankfurt.de

DOI 10.1055/s-0042-103296 VNR2760512016149753976 Dtsch Med Wochenschr 2016; 141: 718-728 (c) Georg Thieme Verlag KG . Stuttgart · New York . ISSN 0012-0472

2:1-AV-Block oder blockierter SV-Bigeminus? I Ein SV-Bigeminus, bei dem die SVES im AV-Knoten blockiert sind, kann einem AV-Block II $^{\circ}$ mit 2:1-Überleitung ähnlich sehen. Entscheidend ist die Bestimmung des Intervalls zwischen übergeleiteten und blockierten P-Wellen: Ist es regelmäßig $( \pm 40 \mathrm{~ms})$, liegt ein AV-Block $\mathrm{II}^{\circ}$ mit 2:1-Überleitung vor. Ist der Abstand von der übergeleiteten zur blockierten P-Welle kürzer als der Abstand von der blockierten zur nächsten übergeleiteten P-Welle (Intervall $<75 \%$ ), liegt ein blockierter SV-Bigeminus vor. Bei diesem handelt es sich nicht um eine Bradykardie.

\section{U-Welle oder AV-Block II ${ }^{\circ}$ mit 2:1-Überleitung? I}

Bei Patienten mit Bradykardie kann eine deutlich sichtbare U-Welle vorliegen, die einer P-Welle ähnlich sehen kann und dann von einem AVBlock $\mathrm{II}^{\circ}$ mit 2:1-Überleitung unterschieden werden muss. Eine U-Welle ist im Gegensatz zu einer P-Welle meist nur in den Brustwandableitungen zu sehen, fast immer breiter als $0 \mathrm{~ms}(160 \mathrm{~ms})$ und kleiner als 0,2 $\mathrm{mV}$ ( $\bullet$ Abb. 10).
Unter Vaguseinfluss nicht-übergeleitete P-Wellen I Moderne Langzeit-EKGs weisen automatisch die längste Pause bzw. niedrigste Herzfrequenz der Registrierperiode aus. Hierbei werden oft isolierte nicht-übergeleitete P-Wellen, die nachts auftreten, dargestellt. Dieses Phänomen ist in der Regel vagal induziert, ohne pathologische Bedeutung und tritt besonders bei Patienten mit neurokardialen Synkopen auf (z.B. Jugendliche, Sportler). Beweisend für eine extrinsische, vagale Ursache ist die Koinzidenz einer oder mehrerer blockierter P-Wellen mit einer gleichzeitig reduzierten Sinusfrequenz: Bei einer pathologischen, infrahisären Leitungsblockierung wird die Überleitung bei reduzierter Sinusfrequenz besser und nur bei Steigerung schlechter $($ Abb. 11).

\section{Konsequenz für Klinik und Praxis}

- Bei der Analyse von Bradykardien sind verschiedene Formen der SinusknotenFunktionsstörungen und der AV-Überleitungsstörung zu unterscheiden.

- Intermittierende Bradykardien gehen typischerweise mit Synkopen einher (z. B. intermittierender Sinusarrest oder AV-Block $\mathrm{III}^{\circ}$ ). Permanente Bradykardien verursachen häufiger Allgemeinsymptome und sind im EKG oft nicht sofort als Ursache der Symptomatik erkennbar (z. B. chronotrope Sinusknoteninkompetenz, sehr langer AV-Block $I^{\circ}$ ).

- Die Dokumentation intermittierender Bradykardien erfordert oft ein LangzeitMonitoring-Verfahren inklusive implantierbare Loop-Rekorder - insbesondere um einen klaren Zusammenhang zwischen einer Symptomatik und der assoziierten Bradykardie zu beweisen.
Vollständiges Literaturverzeichnis unter http://dx.doi.org/10.1055/s-0042-103296

\section{Interessenkonflikt}

Der Autor gibt folgenden Interessenkonflikt an: Er hat Reisekostenerstattung und Vortragshonorare erhalten durch die Firmen Biotronik, Boston-Scientific,

Medtronic, Sorin / Linanova, St. Jude Medical sowie Unterstützung wissenschaftlicher Studien durch die Firmen Biotronik, Boston-Scientific, Medtronic, Sorin/ Linanova und Beratertätigkeit für die Firmen Medtronic, Sorin/Linanova und St. Jude Medical. 
4 Jensen PN, Gronroos NN, Chen LY et al. Incidence of and risk factors for sick sinus syndrome in the general population. J Am Coll Cardiol 2014; 64: 531-538

5 Brignole M, Auricchio A, Baron-Esquivias G et al. 2013 ESC guidelines on cardiac pacing and cardiac resynchronization therapy. Europace 2013; 15: 1070-1118

6 Brignole M, Auricchio A, Baron-Esquivias G et al. 2013 ESC guidelines on cardiac pacing and cardiac resynchronization therapy: Addenda. Eur Heart ]; DOI: 10.1093/eurheartj/eht180

7 Israel CW, Bänsch D, Breithardt O et al. Kommentar zu den neuen ESC-Leitlinien zur Schrittmacher- und kardialen Resynchronisationstherapie. Kardiologe 2015; 9: 35-45 


\section{CME-Fragen}

CME-Teilnahme

- Viel Erfolg bei Ihrer CME-Teilnahme unter http://cme.thieme.de

- Diese Fortbildungseinheit ist 12 Monate online für eine CME-Teilnahme verfügbar.

- Sollten Sie Fragen zur Online-Teilnahme haben, unter http:// cme.thieme.de/hilfe finden Sie eine ausführliche Anleitung.

\section{Welche Aussage zu Bradykardien trifft zu?}

a Eine Bradykardie liegt per Definition nur bei einer Ventrikelfrequenz $<50 /$ min vor.

b Eine Bradykardie ist praktisch immer pathologisch.

c Bradykardien können nur Symptome auslösen, jedoch nie zu einem plötzlichen Herztod führen.

d Jeder AV-Block erfordert eine Herzschrittmacher-Implantation.

e Eine nächtliche Sinusbradykardie von 40-45/ min ist bei einem asymptomatischen Patienten ein Normalbefund.

2. Welche Aussage zum Sick-Sinus-Syndrom trifft nicht zu?

a Eine Form der Sinusknoten-Funktionsstörung ist der intermittierende sinuatriale Block.

b Eine Form der Sinusknoten-Funktionsstörung ist die permanente Sinusbradykardie.

c Eine Form des Sick-Sinus-Syndroms ist der Sinusarrest bei einer Kipptisch-Untersuchung.

d Eine Form der Sinusknoten-Funktionsstörung ist das Brady-TachySyndrom.

e Eine Form der Sinusknoten-Funktionsstörung ist die chronotrope Inkompetenz.

3. Welche Aussage zum AV-Block II ${ }^{\circ}$ trifft zu?

a Beim AV-Block II ${ }^{\circ}$ Typ Wenckebach liegt eine abrupte, unvorhersehbare Blockierung einer einzelnen P-Welle vor.

b Beim AV-Block II ${ }^{\circ}$ Typ Mobitz liegt eine periodische Verlängerung der PQ-Zeit bis zur Blockierung einer einzelnen P-Welle vor.

c Ein 2:1-AV-Block (nur jede 2. P-Welle wird ubergeleitet) stellt immer einen AV-Block II $^{\circ}$ Typ Mobitz und nie einen AV-Block II ${ }^{\circ}$ Typ Wenckebach dar.

d Ein AV-Block II $^{\circ}$ Typ Wenckebach ist meist intranodal lokalisiert (innerhalb des AV-Knotens).

e $\quad$ Ein AV-Block II $^{\circ}$ Typ Mobitz wird unter Karotissinus-Massage höhergradiger.

4. Welche Aussage zum AV-Block bei Vorhofflimmern trifft zu?

a Bei Vorhofflimmern kann ein AV-Block $I^{\circ}$ sicher erkannt werden.

b Bei Vorhofflimmern kann ein AV-Block II ${ }^{\circ}$ Typ Mobitz sicher erkannt werden.

c Bei Vorhofflimmern mit durchgehend regelmäßigem ventrikulärem Rhythmus liegt ein AV-Block III $^{\circ}$ vor.

d Jede Pause bei Vorhofflimmern ist ein AV-Block III'.

e Bei Vorhofflimmern ist oft eine Wenckebach-Periodik der AV-Überleitung zu beobachten.

5. Welche Aussage zu Schenkelblockierungen trifft zu?

a Beim Bild eines Rechtsschenkelblocks liegt in Ableitung V1 eine dominante R-Zacke vor.

b Ein Rechtsschenkelblock ist immer pathologisch.

c Bei einem Rechtsschenkelblock liegt immer ein überdrehter Rechtslagetyp vor.

d Bei einem Linksschenkelblock liegt immer ein überdrehter Linkslagetyp vor.

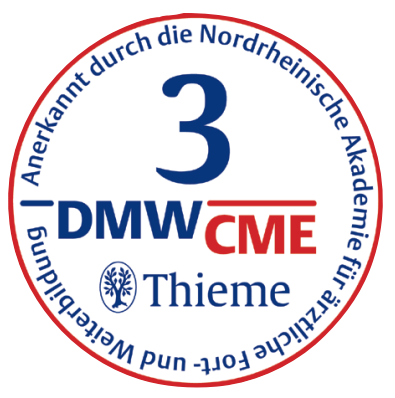

e Beim Bild eines Linksschenkelblocks liegt in Ableitung V6 immer eine dominante R-Zacke vor.

6. Welche der folgenden Schenkelblockierungen ist kein bifaszikulärer Schenkelblock?

a ein kompletter Rechtsschenkelblock

b ein kompletter Linksschenkelblock

c die Kombination Rechtsschenkelblock und linksanteriorer Hemiblock

d die Kombination Rechtsschenkelblock und linksposteriorer Hemiblock

e die Kombination linksposteriorer Hemiblock und septaler Schenkelblock

7. In welcher Phase des Aktionspotenzials des Leitungssystems besteht in der Regel das Problem beim paroxysmalen AV-Block III?

a Phase I

b Phase II

c Phase III

d Phase IV

e keine der genannten

8. Welches EKG-Monitoring ist optimal zur Abklärung von Synkopen, die durchschnittlich alle 3 Monate auftreten?

a Ruhe-EKG

b 24-Stunden-Holter-Monitoring

c 3tägige stationäre Telemetrie

d 14-Tage Event-Rekorder

e implantierbarer Loop-Rekorder

9. Im Rahmen welcher Erkrankung kommt es zu AV-Blockierungen?

a Klinefelter-Syndrom

b Lyme-Disease

c Marfan-Syndrom

d Mitralklappen-Prolaps

e Brugada-Syndrom

10. Bei einem Sportler (Marathonläufer) fallen im Langzeit-EKG 2 einzelne, nachts nicht übergeleitete $P$-Wellen (2:30 Uhr und 2:44 Uhr) auf. Welches ist die wahrscheinlichste Diagnose?

a AV-Block II ${ }^{\circ}$ Typ Mobitz

b $\quad$ AV-Block III $^{\circ}$

c Myokarditis

d Hinterwandinfarkt

e vagal vermittelte nicht übergeleitete P-Welle 


\section{Angaben zur Person}

Name, Vorname, Titel:

Straße, Hausnr.:

PLZ, Ort:

Anschrift: $\square$ privat $\square$ dienstlich

Ich bin Mitglied der Ärztekammer (bitte Namen der Kammer eintragen):

Jahr meiner Approbation:

Ich befinde mich in der Weiterbildung zum:

Ich habe eine abgeschlossene Weiterbildung in ...

(bitte Fach eintragen):

Ich bin tätig als: $\square$ Assistenzarzt $\square$ Oberarzt $\square$ Chefarzt $\square$ niedergelassener Arzt $\square$ Sonstiges Ich bin DMW-Abonnent: $\square$ ja $\square$ nein

Falls nein: ich habe den Fragebogen aus/von:

$\square$ Thieme-connect $\square$ Kollegen $\square$ der Klinik $\square$ einer Bibliothek $\square$ Sonstiges

Lernerfolgskontrolle (Eine Antwort pro Frage ankreuzen)
1. $\square$ A $\square$ B $\square$ C $\square$ D $\quad \square \mathrm{E}$
2. $\square \mathrm{A} \quad \square \mathrm{B} \quad \square \mathrm{C} \quad \square \mathrm{D} \quad \square \mathrm{E}$
3. $\square$ A $\square$ B $\square$ C $\square$ D $\quad \square \mathrm{E}$
4. $\square$ A $\square$ B $\square$ C $\square$ D $\square$ E
5. $\square$ A $\square$ B $\square$ C $\square$ D $\square$ E
6. $\square$ A $\square$ B $\square$ C $\square$ D $\square$ E
7. $\square$ A $\square$ B $\square \mathrm{C} \quad \square$ D $\square$ E
8. $\square$ A $\square$ B $\square$ C $\square$ D $\square \mathrm{E}$
9. $\square \mathrm{A} \square \mathrm{B} \quad \square \mathrm{C} \quad \square \mathrm{D} \quad \square \mathrm{E}$
10. $\square \mathrm{A} \quad \square \mathrm{B} \quad \square \mathrm{C} \quad \square \mathrm{D} \quad \square \mathrm{E}$

Ich versichere, dass ich die Beantwortung der Fragen selbst und ohne fremde Hilfe durchgeführt habe

Ort, Datum:

Unterschrift:

Bitte in dieses Feld Ihre DMW Abonnement-Nummer eintragen:

\section{Fragen zur Zertifizierung}

1. Das Thema des Beitrages kommt in meiner ärztlichen Tätigkeit $\square$ häufig vor $\square$ selten vor $\square$ regelmäßig vor $\square$ gar nicht vor

2. Bei diesem Thema habe ich $\square$ eine feste Gesamtstrategie $\square$ keine Strategie $\square$ noch offene Einzelprobleme

3. In Bezug auf das Thema des Beitrages

$\square$ fühle ich mich nach dem Studium des Beitrags in meiner Strategie bestätigt

$\square$ habe ich meine Strategie verändert:

$\square$ habe ich erstmals eine einheitliche Strategie erarbeitet

$\square$ habe ich keine einheitliche Strategie ableiten können

4. Wurden aus der Sicht Ihrer täglichen Praxis heraus wichtige Aspekte des Themas

nicht erwähnt: $\square$ ja, welche $\square$ nein

zu knapp abgehandelt? $\square$ ja, welche $\square$ nein

überbewertet? $\square$ ja, welche $\square$ nein

5. Verständlichkeit des Beitrages

$\square$ Der Beitrag ist nur für Spezialisten verständlich

$\square$ Der Beitrag ist auch für Nicht-Spezialisten verständlich

6. Beantwortung der Fragen

$\square$ Die Fragen lassen sich aus dem Studium des Beitrags allein beantworten

$\square$ Die Fragen lassen sich nur unter Zuhilfenahme zusätzlicher Literatur beantworten

7. Die Aussagen des Beitrages benötigen eine ausführlichere Darstellung

$\square$ zusätzlicher Daten

$\square$ von Befunden bildgebender Verfahren

$\square$ die Darstellung ist ausreichend

8. Wieviel Zeit haben Sie für das Lesen des Beitrages und der Bearbeitung des Quiz benötigt?

Zertifizierungsfeld (wird durch die DMW ausgefüllt)

Ihr Ergebnis:

Sie haben von 10 Fragen richtig beantwortet.

Sie haben $\square$ bestanden und 3 Punkte erworben $\square$ nicht bestanden $\square$ ungültig, weil:

Stuttgart, den Stempel/Unterschrift 